\title{
Portable Electrochemical Immunochromatography Platform for Point-of-care Testing and Comparison with Enzyme-linked Immunosorbent Assay
}

\author{
Wataru Iwasaki, ${ }^{1 *}$ Nobutomo Morita, ${ }^{1}$ and Masaya Miyazaki ${ }^{2 \dagger}$ \\ ${ }^{1}$ Sensing System Research Center, National Institute of Advanced Industrial Science and Technology, \\ 807-1 Shuku-machi, Tosu, Saga 841-0052, Japan \\ ${ }^{2}$ Center of Plasma Nano-interface Engineering, Kyushu University, \\ 744 Motooka, Nishi-ku, Fukuoka 819-0395, Japan
}

(Received April 2, 2020; accepted June 17, 2020)

Keywords: electrochemical measurement, immunochromatography, paper-based analytical device, point-of-care testing

We conducted electrochemical immunochromatography using a developed electrochemical immunochromatography platform. We used immunoglobulin $\mathrm{G}$ ( $\operatorname{IgG}$ ) as a representative protein. We also conducted enzyme-linked immunosorbent assay (ELISA) as a control test. We used the same reagents except for the substrate and compared their limits of detection (LODs) and detection ranges. We succeeded in measuring $\operatorname{IgG}$ concentrations ranging from $30 \mathrm{ng} / \mathrm{ml}$ to $10 \mu \mathrm{g} / \mathrm{ml}$ and obtained $23.00 \mathrm{ng} / \mathrm{ml}$ as the LOD for electrochemical immunochromatography. The detection signal was not saturated even at $10 \mu \mathrm{g} / \mathrm{ml}$, so it seems that higher concentrations of IgG can also be measured. The LOD was approximately 80 -fold higher than that of ELISA $(0.30 \mathrm{ng} / \mathrm{ml})$; however, the detection range was one order of magnitude higher than that of ELISA. While electrochemical immunochromatography is less sensitive than ELISA, we demonstrated sufficient sensitivity for its use in a point-of-care testing (POCT) device. Furthermore, this information will be very helpful when introducing an assay system already established in ELISA to POCT.

\section{Introduction}

Point-of-care testing (POCT) is an easy, fast, and low-cost diagnostic method conducted at the bedside, near the patient, and on-site. ${ }^{(1)}$ A portable glucose sensor is one of the representative diagnostic devices for POCT. ${ }^{(2,3)}$ It enables diabetic and pre-diabetic patients to measure and control their blood glucose concentration by themselves. Immunochromatography is also one of the diagnostic methods for biomarkers such as human chorionic gonadotropin (hCG) in urine for a pregnancy test. ${ }^{(4)}$ It had been applied to various diagnoses because of its simple use, short measurement time of tens of minutes, and its low cost of less than 10 dollars per measurement. However, it is not quantitative and it is difficult to achieve a low detection limit. A microfluidic

\footnotetext{
*Corresponding author: e-mail: wataru.iwasaki@aist.go.jp

†Present address: HaKaL inc., 6-27 Nishi-nakasu, Chuo-ku, Fukuoka 810-0002, Japan https://doi.org/10.18494/SAM.2020.2897
} 
paper-based analytical device ( $\mu$ PAD) was developed as a new POCT technique in 2007. ${ }^{(5)}$ It uses paper as the substrate for the immunoassay. $\mu$ PADs have many advantages such as low-cost measurement and easy fabrication. Therefore, they have attracted much attention and been studied by several groups in the last decade. Many $\mu$ PADs based on, for example, colorimetric, fluorescent, and electrochemical methods have already been developed. ${ }^{(6-8)}$ Electrochemical immunochromatography, in which electrochemical methods are introduced into immunochromatography, is anticipated to be used in standard $\mu$ PADs. Therefore, we previously developed an electrochemical immunochromatography platform to investigate the effects of the contact pressure and flow rate between an electrode and a nitrocellulose membrane. ${ }^{(9-11)}$ Usually, electrodes are printed on a paper when an electrochemical method is introduced into $\mu$ PADs. ${ }^{(7,12,13)}$ In this case, the electrodes are disposable along with the paper, increasing their cost of use. Another problem is that the printed electrode fills the porous membrane and obstructs the flow by capillary force. Our device has separate electrodes and membranes. The electrodes fabricated by sputtering gold have a flat surface, which is important for sensitive detection. In this study, we used this electrochemical immunochromatography platform to attempt an immunoanalysis of a representative protein, immunoglobulin $G(\operatorname{IgG})$, and investigated the detectability by electrochemical immunochromatography. As a control test, we performed enzyme-linked immunosorbent assay (ELISA), a common immunological analysis method, to compare the detection capability. When constructing a new immunoassay system, it is necessary to consider the combination of primary and secondary antibodies and antigens, and it is difficult to predict the level of detection capability that can be obtained. Since ELISA has already become a standard method for many antigens, and data on their combinations and detectability have been established, the comparison of the detectability by electrochemical immunochromatography and ELISA in this study is expected to be applied to the construction of assay systems for applying various antigens to electrochemical immunochromatography in the future.

\section{Materials and Methods}

\subsection{Reagents and apparatus}

A nitrocellulose membrane (HF120, Merck Millipore Co. Ltd., Massachusetts, USA) was used as an immunochromatographic strip for electrochemical immunochromatographic assay. Anti-rabbit IgG antibody (111-005-046, Jackson ImmunoResearch Laboratories Inc., PA, USA), rabbit IgG (AB-105-C, R\&D Systems, Inc., NE, USA), and alkaline-phosphatase-labeled antirabbit IgG antibody (AM08098AP-N, OriGene Technologies, Inc., MD, USA) were used for sandwich immunoassay of rabbit IgG as the primary antibody, antigen, and secondary antibody, respectively. $p$-Aminophenylphosphate monosodium salt ( $p$ APP, A5030, LTK Laboratories, Inc., MN, USA) and $p$-nitrophenylphosphate ( $p$ NPP, 37621, Thermo Fisher Scientific K.K., Tokyo, Japan) were used as substrates for the electrochemical method and colorimetric method, respectively. SuperBlock ${ }^{\mathrm{TM}}$ T20 (TBS) Blocking Buffer (Thermo Fisher Scientific K.K.) was used to block the nitrocellulose membrane. An ALS630D electrochemical analyzer (BAS Inc., 
Tokyo, Japan) was used for electrochemical measurement. An iMark ${ }^{\mathrm{TM}}$ microplate reader (BioRad Laboratories, Inc., CA, USA) was used for ELISA.

\subsection{Electrochemical immunochromatography platform}

For the electrochemical detection of IgG, we used our previously developed electrochemical immunochromatography platform. ${ }^{(10,11)}$ The platform consists of a nitrocellulose membrane, absorbent pad, electrochemical detector, and two polymethyl methacrylate (PMMA) plates (Fig. 1). The electrochemical detector was fabricated by standard photolithography and the liftoff process as described in a previous work. ${ }^{(10)}$ The nitrocellulose membrane was cut to a width of $5 \mathrm{~mm}$ and a length of $50 \mathrm{~mm}$. The electrochemical detector and the absorbent pad were attached to the nitrocellulose membrane at the center and an edge, respectively. The contact condition between the nitrocellulose membrane and the electrochemical detector is important. ${ }^{(9)}$ Therefore, the structure is designed to stabilize the contact state by tightening with a controlled torque and pressing with polydimethylsiloxane (PDMS). The top PMMA plate has an inlet upstream of the nitrocellulose membrane.

\subsection{Electrochemical immunochromatography measurement}

We prepared an immunochromatographic strip before electrochemical immunochromatography measurement. The primary antibody was immobilized on an immunochromatographic strip by the dropwise addition of $2 \mu 1$ of primary antibody solution ( $5 \mathrm{mM}$ phosphate buffer of $\mathrm{pH} 7.3$ containing $1 \mathrm{mg} / \mathrm{ml}$ of primary antibody) on a nitrocellulose membrane at a distance of $20 \mathrm{~mm}$ from the upstream edge, and then the membrane was incubated at $37{ }^{\circ} \mathrm{C}$ for $2 \mathrm{~h}$. After that, the membrane was immersed in a blocking buffer, and it was shaken for 15 min with a shaker. Next, it was washed by immersing and shaking in $20 \mathrm{mM}$ TBST for $30 \mathrm{~min}$. The membrane was dried at $4{ }^{\circ} \mathrm{C}$ overnight. Electrochemical immunochromatography was conducted using the prepared immunochromatographic strip as follows (Fig. 2). First, $10 \mu \mathrm{l}$ of IgG solution was added to the inlet of the electrochemical

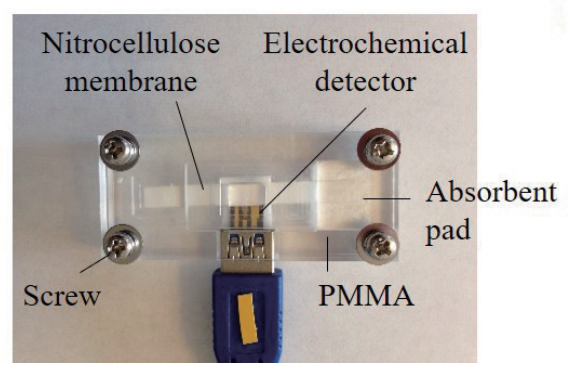

(a)

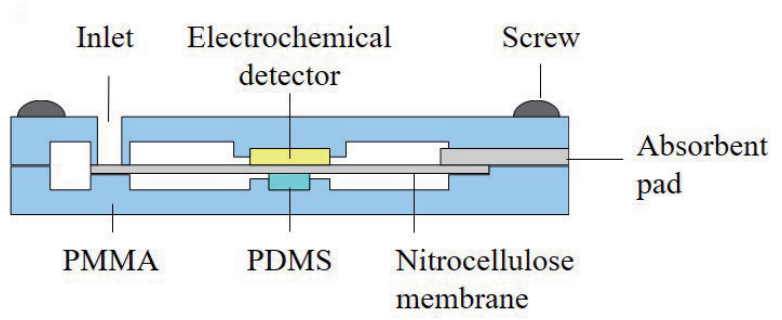

(b)

Fig. 1. (Color online) Electrochemical immunochromatography platform. (a) Top-view photograph. ${ }^{(10)}$ (b) Schematic cross-sectional view. 

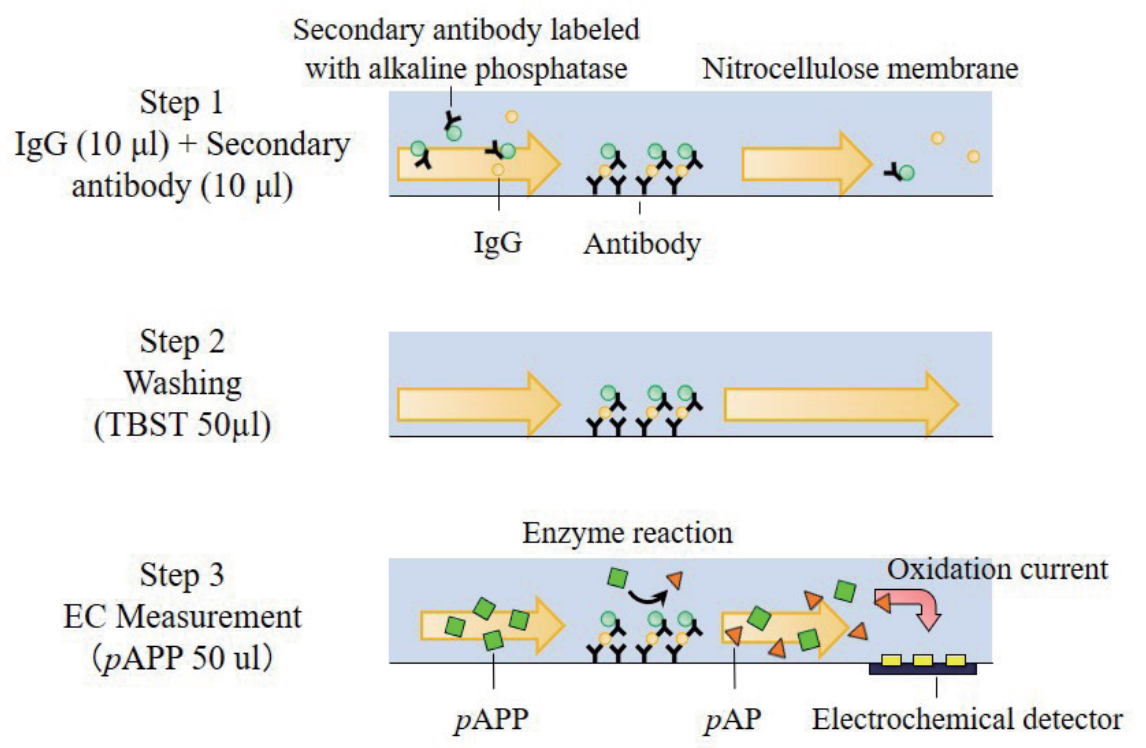

Fig. 2. (Color online) Measurement procedure of electrochemical immunochromatography.

immunochromatography platform, then $10 \mu \mathrm{l}$ of secondary antibody solution diluted 500-fold with TBST containing $0.1 \%$ BSA was immediately added to the inlet. The concentrations of IgG used were $30,100,300,1000,3000$, and $10000 \mathrm{ng} / \mathrm{ml}$. IgG and secondary antibody were flowed in turn through the membrane in the downstream direction by capillary force. IgG was captured by the immobilized primary antibody, and the secondary antibody captured the IgG. Then sandwiched IgG conjugate was formed in the area of the immobilized primary antibody. Untrapped IgG and secondary antibodies were flushed downstream by adding $50 \mu 1$ of washing buffer (20 mM TBST of pH 7.4 containing $0.05 \%$ Tween20) to the inlet. After that, $50 \mu \mathrm{l}$ of $20 \mathrm{mM}$ TBST of $\mathrm{pH} 8.0$ containing $1 \mathrm{mg} / \mathrm{ml}$ of $p$ APP and $50 \mathrm{mM} \mathrm{KCl}$ was added to the inlet. The $p$ APP reacted with alkaline phosphatase captured in the area of the immobilized primary antibody and produced $p$-aminophenol ( $p \mathrm{AP})$. The produced $p \mathrm{AP}$ flowed downstream and was detected at the electrochemical detector. We conducted each measurement three times.

\subsection{ELISA}

We performed ELISA as a control test. The entire procedure was performed at room temperature. IgG solutions with various concentrations $(0.1,0.3,1,3,10,30,300,1000,3000$, and $10000 \mathrm{ng} / \mathrm{ml}$ ) diluted with PBS were prepared. First, $100 \mu \mathrm{l}$ of primary antibody solution (PBS containing $10 \mu \mathrm{g}$ of primary antibody per $\mathrm{ml}$ ) was added to wells of a microplate and incubated for $1 \mathrm{~h}$. Next, $200 \mu \mathrm{l}$ of PBS containing 1.0\% BSA was added to the wells following washing with $200 \mu \mathrm{l}$ of PBST three times, then incubated for $2 \mathrm{~h}$. Each well was washed again with $200 \mu \mathrm{l}$ of PBST three times. Then, $100 \mu \mathrm{l}$ of the $\mathrm{IgG}$ solutions with different concentrations were added to the wells and incubated for $1 \mathrm{~h}$. $100 \mu \mathrm{l}$ of secondary antibody solution diluted 4000 -fold with TBS was added to the wells following washing with $200 \mu 1$ of TBST three 
times, and then incubated for $1 \mathrm{~h}$. Each well was washed again with $200 \mu \mathrm{l}$ of TBST three times. Next, $100 \mu \mathrm{l}$ of pNPP solution was added to the wells. After $30 \mathrm{~min}$, we stopped the enzyme reaction of alkaline phosphatase and pNPP by adding $100 \mu \mathrm{l}$ of $2 \mathrm{~N} \mathrm{NaOH}$ solution. We measured the absorbance of each well at $405 \mathrm{~nm}$.

\section{Results and Discussion}

Figure 3(a) shows a chronoamperogram of each concentration obtained by electrochemical immunochromatography. We started chronoamperometry just after pAPP was added to the inlet. The applied potential was $0.15 \mathrm{~V}$ vs $\mathrm{Ag} / \mathrm{AgCl}$. The electrochemical signal rapidly increased after $100 \mathrm{~s}$. This means that pAP reached the electrochemical detector. After that, a stable signal was observed. We plotted the electrochemical current at $250 \mathrm{~s}$ for each concentration [Fig. 3(b)], where each plot was the average of triplicate measurements. Since

(a)

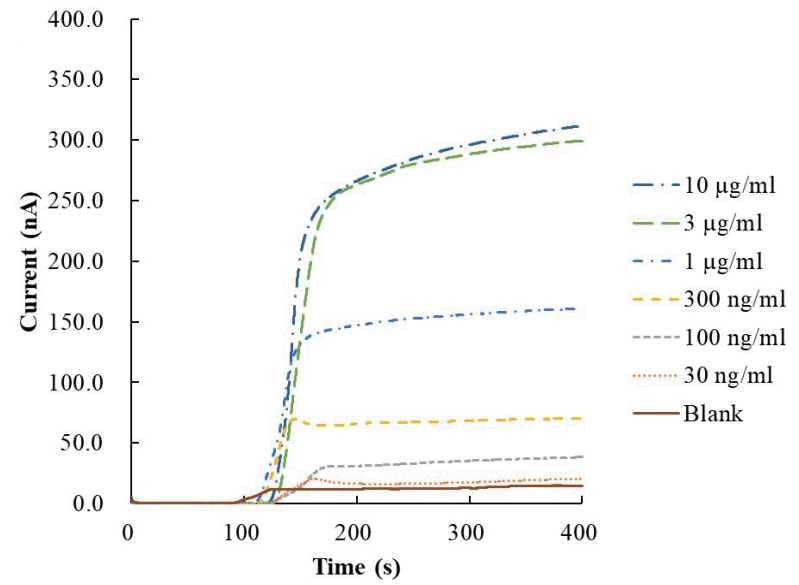

(b)

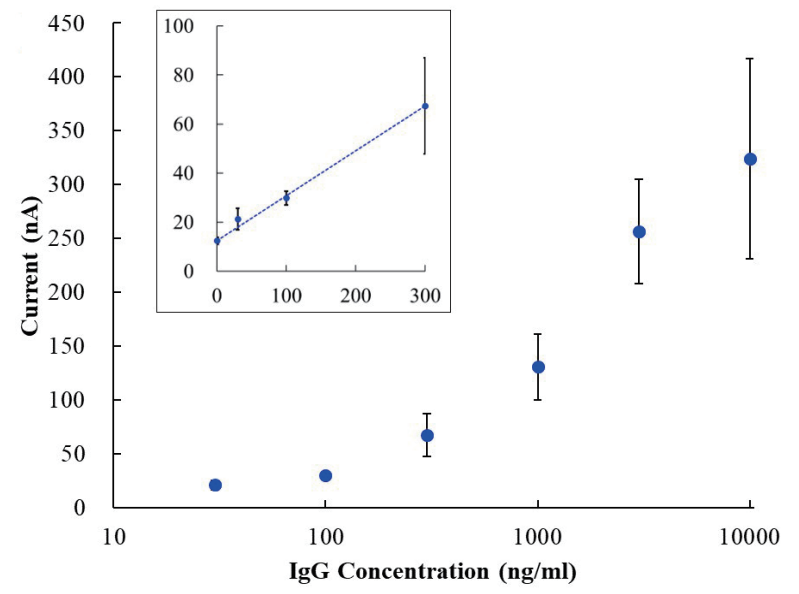

Fig. 3. (Color online) Results of electrochemical immunochromatography measurement for IgG. (a) Chronoamperogram. The applied potential was $0.15 \mathrm{~V}$ vs $\mathrm{Ag} / \mathrm{AgCl}$. (b) Calibration curve $(n=3)$. The inset of (b) is a magnified view of the range from 0 to $300 \mathrm{ng} / \mathrm{ml}$. Error bars represent standard deviations. 
the signals from the electrochemical measurements did not show a sigmoidal curve and did not reach an upper limit, it is assumed that the upper limit of the detection range is even higher than $10000 \mathrm{ng} / \mathrm{ml}$. However, electrochemical immunochromatography may not be suitable for measurements at high concentrations because it shows a large deviation at high concentrations. On the other hand, it shows a very small deviation at low concentrations. The electrochemical signal of the blank was $12.46 \pm 1.40 \mathrm{nA}$. The limit of detection (LOD) could not be calculated using a calibration curve fitted with a sigmoidal function due to the small signal of the blank. Because of the high linearity in the low concentration range, the LOD was calculated to be $23.00 \mathrm{ng} / \mathrm{ml}$ using a linear approximation line from 0 to $300 \mathrm{ng} / \mathrm{ml}$ (intercept $=$ blank) and blank $+3 \sigma$. We also performed ELISA as a control test. Figure 4 shows the result of ELISA. We succeeded in obtaining a calibration curve with a small variation. As with electrochemical immunochromatography, the LOD could not be obtained from the sigmoid curve because of the small blank values in ELISA. A linear approximation line from 0 to $100 \mathrm{ng} / \mathrm{ml}$ (intercept $=$ blank) was used to obtain $0.30 \mathrm{ng} / \mathrm{ml}$ for the LOD. Electrochemical immunochromatography had an approximately 80 -fold higher LOD than ELISA. The absorbance was saturated at a concentration of $1 \mu \mathrm{g} / \mathrm{ml}$ in ELISA, but electrochemical immunochromatography seemed to be able to measure concentrations above $10 \mu \mathrm{g} / \mathrm{ml}$. Thus, electrochemical immunochromatography seems to be able to analyze a wider range of concentrations than ELISA. It is considered that one of the main reasons for this is the difference in the amounts of the sample and the reagents. The amounts of antibody, antigen, and secondary antibody used in our electrochemical immunochromatography were 2,1/10, and 4/5 times those in ELISA, respectively. The LOD may have been lower for electrochemical immunochromatography because of the lower antigen content. In addition, the short reaction time may have reduced the amount of antigen trapped by the antibody. If a slower-flowing nitrocellulose membrane is used, the analysis time will be longer, but the detection limit will be improved. Similarly, higher concentrations

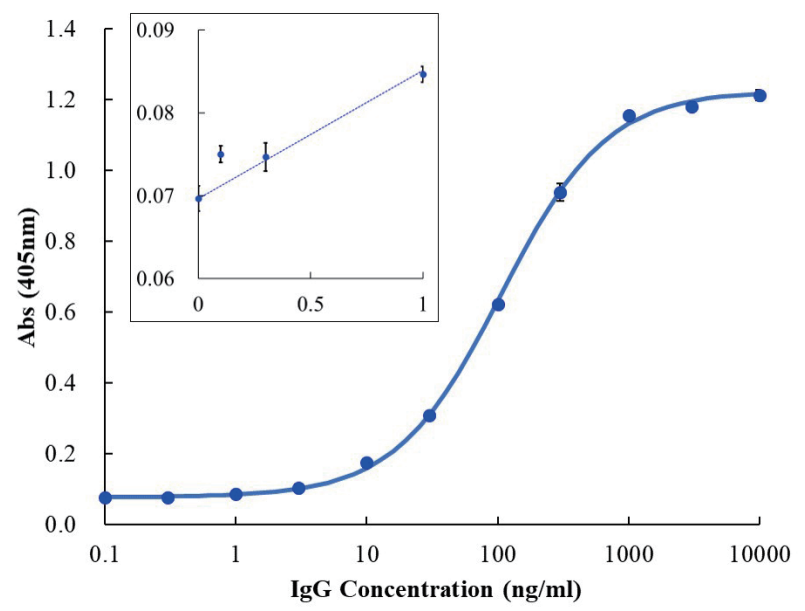

Fig. 4. (Color online) Calibration curve obtained by ELISA $(n=3)$. The inset is a magnified view of the range from 0 to $1 \mathrm{ng} / \mathrm{ml}$. Error bars represent standard deviations. 
could be measured without saturation in the case of electrochemical immunochromatography, even with the same concentration of antigens, because of the small amount of antigens. The amount of antibodies was also higher in electrochemical immunochromatography, which may have contributed to the higher detection limit. The sensitivity of electrochemical immunochromatography could be further improved by adopting a nanocarbon film electrode, which shows lower background noise. ${ }^{(14)}$ While electrochemical immunochromatography is less sensitive than ELISA, ELISA takes about $3 \mathrm{~h}$ to perform after preparing the blocked antibody immobilized plates, whereas electrochemical immunochromatography can be performed in about 20 min under similar conditions. An important factor determining the analysis time is the diffusion distance. The amount of reaction in various reactions (e.g., antigen-antibody reactions, enzymatic reactions) depends on the frequency of molecular collisions. The shorter the diffusion distance, the greater the probability of materials colliding with each other and the faster the reaction. In the present system, the diffusion distance of ELISA is $2.60 \mathrm{~mm}$, whereas the diffusion distance of immunochromatography is $6 \mu \mathrm{m}$, which is half of the nominal pore size ${ }^{(15)}$ a difference by a factor of about 430 . Therefore, the reaction time can be dramatically reduced by using immunochromatography. It is also a superior method for POCT because it requires much less manual work than ELISA. The information provided in the paper on the difference in the LOD and the detection range will be very useful when applying ELISA to electrochemical immunochromatography.

\section{Conclusions}

We measured $\operatorname{IgG}$ as a representative protein using a developed electrochemical immunochromatography platform and achieved an LOD of $23.00 \mathrm{ng} / \mathrm{ml}$. Although the LOD was approximately 80 -fold higher than that of ELISA, the detection range was one order of magnitude higher than that of ELISA. This information will be very helpful when introducing an assay system already established in ELISA to POCT. In this study, we used a desktop PC and a desktop potentiostat, but it is possible to construct a portable system by using a USB-type potentiostat and a tablet PC already available on the market. Such a portable system is expected to be used as a POCT device for quantitative measurement.

\section{References}

1 A. Warsinke: Anal. Bioanal. Chem. 393 (2009) 1393. https://doi.org/10.1007/s00216-008-2572-0

2 E. H. Yoo and S. Y. Lee: Sensors 10 (2010) 4558. https://doi.org/10.3390/s100504558

3 D. R. Matthews, E. Bown, A. Watson, R. R. Holman, J. Steemson, S. Hughes, and D. Scott: Lancet 1 (1987) 778. https://doi.org/10.1016/s0140-6736(87)92802-9

4 G. A. Posthuma-Trumpie, J. Korf, and A. van Amerongen: Anal. Bioanal. Chem. 393 (2009) 569. https://doi. org/10.1007/s00216-008-2287-2

5 A. W. Martinez, S. T. Phillips, M. J. Butte, and G. M. Whitesides: Angewandte Chemie International Edition 46 (2007) 1318. https://doi.org/10.1002/anie.200603817

6 C. M. Cheng, A. W. Martinez, J. L. Gong, C. R. Mace, S. T. Phillips, E. Carrilho, K. A. Mirica, and G. M. Whitesides: Angew. Chem.-Int. Edit. 49 (2010) 4771. https://doi.org/10.1002/anie.201001005

7 Z. H. Nie, F. Deiss, X. Y. Liu, O. Akbulut, and G. M. Whitesides: Lab Chip 10 (2010) 3163. https://doi. org $/ 10.1039 / \mathrm{c} 01 \mathrm{c} 00237 \mathrm{~b}$

8 A. K. Yetisen, M. S. Akram, and C. R. Lowe: Lab Chip 13 (2013) 2210. https://doi.org/10.1039/c31c50169h 
9 W. Iwasaki, R. R. Sathuluri, O. Niwa, and M. Miyazaki: Anal. Sci. 31 (2015) 729. https://doi.org/10.2116/ analsci.31.729

10 W. Iwasaki, R. R. Sathuluri, R. Kurita, O. Niwa, and M. Miyazaki: Sens. Mater. 28 (2016) 1329. https://doi. org/

11 W. Iwasaki, C. Kataoka, K. Sawadaishi, K. Suyama, N. Morita, and M. Miyazaki: Sensors 20 (2020) 4781. https://doi.org/10.3390/s20174781

12 A. Apilux, W. Dungchai, W. Siangproh, N. Praphairaksit, C. S. Henry, and O. Chailapakul: Anal. Chem. 82 (2010) 1727. https://doi.org/10.1021/ac9022555

13 M. Pavithra, S. Muruganand, and C. Parthiban: Sens. Actuator B-Chem. 257 (2018) 496. https://doi. org/10.1016/j.snb.2017.10.177

14 O. Niwa, J. Jia, Y. Sato, D. Kato, R. Kurita, K. Maruyama, K. Suzuki, and S. Hirono: J. Am. Chem. Soc. 128 (2006) 7144. https://doi.org/10.1021/ja0606091

15 J. Li, D. McMillan and J. Macdonald: Sens. Mater. 27 (2015) 549. https://doi.org/10.18494/SAM.2015.1147 